\title{
Video Article \\ Evaluation of the Feasibility, Safety, and Accuracy of an Intraoperative High- intensity Focused Ultrasound Device for Treating Liver Metastases
}

\author{
Aurélien Dupre ${ }^{1,2}$, David Melodelima ${ }^{2}$, David Perol ${ }^{3}$, Yao Chen ${ }^{1}$, Jérémy Vincenot ${ }^{2}$, Jean-Yves Chapelon ${ }^{2}$, Michel Rivoire $^{1,2}$ \\ ${ }^{1}$ Department of Surgical Oncology, Centre Léon Bérard \\ ${ }^{2}$ Applications des ultrasons à la thérapie (LabTAU), Institut national de la santé et de la recherche médicale (INSERM), Centre Léon Bérard, Université Lyon 1, \\ Université Lyon \\ ${ }^{3}$ Biostatistics and Treatment Evaluation Unit, Centre Léon Bérard
}

Correspondence to: Aurélien Dupre at aurelien.dupre@lyon.unicancer.fr, David Melodelima at David.Melodelima@inserm.fr

URL: https://www.jove.com/video/57964

DOI: doi:10.3791/57964

Keywords: Cancer Research, Issue 143, High-intensity focused ultrasound, HIFU, ultrasound, liver metastases, colorectal cancer, thermal ablation, focal destruction, clinical trial

Date Published: 1/9/2019

Citation: Dupre, A., Melodelima, D., Perol, D., Chen, Y., Vincenot, J., Chapelon, J.Y., Rivoire, M. Evaluation of the Feasibility, Safety, and Accuracy of an Intraoperative High-intensity Focused Ultrasound Device for Treating Liver Metastases. J. Vis. Exp. (143), e57964, doi:10.3791/57964 (2019).

\section{Abstract}

Today, the only potentially curative option in patients with colorectal liver metastases is surgery. However, liver resection is feasible in less than $20 \%$ of patients. Surgery has been widely used in association with radiofrequency, cryotherapy, or microwaves to expand the number of treatments performed with a curative intent. Nevertheless, several limitations have been documented when using these techniques (i.e., a traumatic puncture of the parenchyma, a limited size of lesions, and an inability to monitor the treatment in real-time).High-intensity focused ultrasound (HIFU) technology can achieve precise ablations of biological tissues without incisions or radiation. Current HIFU devices are based on an extracorporeal approach with limited access to the liver. We have developed a HIFU device designed for intraoperative use. The use of a toroidal transducer enables an ablation rate $\left(10 \mathrm{~cm}^{3} \cdot \mathrm{min}^{-1}\right)$ higher than any other treatment and is independent of perfusion.

The feasibility, safety, and accuracy of intraoperative HIFU ablation were evaluated during an ablate-and-resect prospective study. This clinical phase I and Ila study was performed in patients undergoing hepatectomy for liver metastases. The HIFU treatment was performed on healthy tissue scheduled for resection.Liver metastases measuring less than $20 \mathrm{~mm}$ will be targeted during phase Ilb (currently ongoing). This set-up allows the real-time evaluation of HIFU ablation while protecting participating patients from any adverse effects related to this new technique.

Fifteen patients were included in phase I-Ila and 30 HIFU ablations were safely created within $40 \mathrm{~s}$ and with a precision of 1-2 mm. The average dimensions of the HIFU ablations were $27.5 \times 21.0 \mathrm{~mm}^{2}$, corresponding to a volume of approximately $7.5 \mathrm{~cm}^{3}$. The aim of the ongoing phase IIb is to ablate metastases of less than $20 \mathrm{~mm}$ in diameter with a $5 \mathrm{~mm}$ margin.

\section{Video Link}

The video component of this article can be found at https://www.jove.com/video/57964/

\section{Introduction}

Colorectal liver metastases (CLMs) represent a major public health issue. When resection and/or ablation of CLM can be achieved, a cure is possible, and five-year survival rates of up to $51 \%$ have been reported ${ }^{1}$. Despite progress in surgery (complex surgical techniques) ${ }^{2}$ and downsizing obtained with chemotherapy ${ }^{3}$, only a minority of patients are eligible for surgery (less than $20 \%$ ). When metastases are confined to the liver, surgery is precluded by the size and/or location of metastases, their number, or because the necessary resection will leave an insufficient volume of functional liver ${ }^{4}$.

Radiofrequency ablation (RFA) and microwave ablation (MWA) have been widely used in association with surgery to increase the number of patients treated with a curative intent. However, these techniques require the intraparenchymal introduction of a probe. Moreover, these techniques are inadequate due to the heat-sink effect of blood flow, and real-time monitoring is not possible with these techniques.

Medical ultrasound is mainly known as a diagnostic imaging technique. However, ultrasound can be used as a modality to deliberately cause tissue damage. The energy of the HIFU beam is several orders of magnitude greater than those of a standard diagnostic ultrasound beam. The high energy levels carried in a HIFU beam are magnified further by focusing the beam deep in tissues with precision to a small volume while sparing surrounding tissues. The tissue temperature at the focal point quickly rises to $70-90{ }^{\circ} \mathrm{C}$, resulting in an irreversible destruction by coagulative necrosis $^{5}$. HIFU has been proven effective in a wide range of clinical applications, especially prostate cancer ${ }^{6,7}$. Current medical devices using HIFU are based on an extracorporeal approach, and the single ablation achieved is small and ellipsoidal. The dimensions of every single lesion vary according to the transducer characteristics but are typically $1-3 \mathrm{~mm}$ (transverse) and $8-15 \mathrm{~mm}$ (along the beam axis) ${ }^{8}$. To treat tumors, hundreds of lesions must be placed systematically side by side to ablate the target tumor and some of the surrounding normal tissue margin. The placement of small lesions side by side requires precision that is difficult to ensure in moving organs and results in long-lasting 
treatments (in the order of $1-2 \mathrm{~h}$ ). Whereas numerous publications have reported about MWA and RFA for the treatment of CLM ${ }^{9,10,11,12,13}$ few studies have been published about HIFU for CLM ${ }^{14,15}$. HIFU treatment presents several potential advantages when compared to MWA or RFA as follows: there is no need to puncture the parenchyma, the treatment is independent of perfusion ${ }^{8}$, and real-time monitoring is possible. Extracorporeal treatment of the liver is difficult because the focusing effect is altered by the respiratory motion of the liver and the presence of the ribcage, which may stop the propagation of ultrasound waves.

As focal destruction is often associated with open liver surgery in the management of patients with CLM, we aimed to design a new and powerful HIFU. Such a device would be able to perform large ablations in a short amount of time. Thus, we developed a new technology based on toroida transducers ${ }^{16}$ with encouraging results in preliminary in vitro and preclinical work ${ }^{17,18,19}$. We previously demonstrated in a porcine model that this HIFU device can create reproducible ablations in $40 \mathrm{~s}^{20}$. The average volume of these ablations was $7 \mathrm{~cm}^{3}$, corresponding to an average diameter of $20 \mathrm{~mm}$ and an average long axis of $25 \mathrm{~mm}$. These ablations were created by activating each emitter with the same phase and by applying an acoustic power of $70 \mathrm{~W}$ (free-field acoustic power) for $40 \mathrm{~s}^{18}$. To confirm these results, this HIFU device was used in a controlled trial in patients undergoing hepatectomy for CLM. The objective of the study was to assess the safety and the feasibility of the procedure. Data regarding the efficacy and the accuracy of the procedure were also collected. This HIFU device was not developed to replace the resection part of surgery. The long-term objective is to provide a complementary tool to surgeons in order to expand the number of patients treated with a curative intent by treating otherwise unresectable patients, using HIFU.

The study participants could not expect any personal benefit from their participation, and the priority was to avoid any loss of chance, which is why an ablate-and-resect study was designed. The surgical procedure had to be identical to the one patients would have undergone if not included in the study. For this first use of the HIFU device in humans, ablations were created on the part of the liver scheduled for resection, in order to protect participating patients from any adverse effects related to the use of this new device.

\section{Study Design:}

The protocol was a prospective, single-center phase I/II study designed to assess specific objectives in three different phases (I, Ila, and Ilb). In phases I and Ila, the HIFU treatment was performed in parts of the liver planned for resection but at a distance from metastases. Liver metastases were targeted during phase $\mathrm{Ilb}$. Data of phase $\mathrm{llb}$ are not presented as this part of the protocol is still ongoing.

The primary objectives of phase I were to evaluate the safety, feasibility, and tolerability of HIFU ablation for patients with CLM. The safety and feasibility were assessed by the ability to use the HIFU device in aseptic conditions. The ability to perform two HIFU ablations without injuring organs adjacent to the liver was also assessed. In all patients, one HIFU lesion was superficial and one was created with the focal zone placed at least $1 \mathrm{~cm}$ deeper. Vital signs were monitored during and for $5 \mathrm{~min}$ after the HIFU ablations in order to assess the tolerability of the procedure.

Secondary objectives were to determine the reliability of the ultrasound images and the ease of use of the device. The reliability of the ultrasound images was assessed by using the imaging probe integrated with the HIFU device to visualize and target the region to be treated. Ease of use was assessed by the ability to place the HIFU device such that ablations could be performed in at least $80 \%$ of the whole liver. The entire HIFU procedure has to be performed in less than 30 min.

This two-step phase was designed following Lee's sequential criteria ${ }^{21}$, allowing an early termination of enrollment in the case of an unacceptable global failure rate. The definition of failure was a period longer than 30 min to perform the HIFU procedure, the inability to place the focal zone at least $1 \mathrm{~cm}$ from Glisson's capsule, asepsis failure when using the device, HIFU lesions in adjacent organs, changes in vital signs superior to $10 \%$ from the baseline. The enrollment was considered to be closed if a failure was observed in at least $2 / 2$ patients (first step) and $3 / 6$ patients (second step).

In phase Ila, a major anatomical structure was mimicked by a metallic marker with a diameter of $5 \mathrm{~mm}$, implanted in the liver parenchyma. The primary objective of phase lla was to assess the accuracy of the HIFU device. In step 1, the ability to target the metallic marker with safety margins was assessed. In step 2, the ability to ablate the liver at a predetermined distance from the metallic marker was assessed. The ideal distance between the metallic marker and the closest ablation boundary was fixed at $7.5 \mathrm{~mm}$, with a tolerated range of 1-15 mm. The secondary objective was to assess the safety of the procedure by creating HIFU lesions in the liver without injury to adjacent organs. The feasibility to image HIFU ablations using the integrated ultrasound imaging probe was a common secondary endpoint with phase I. Three to six patients were included in both steps of phase Ila. Binomial probabilities in 3 and 6 patients were used. If a failure occurred in at least $54 \%$ and $32 \%$ of the patients, respectively, there was a $90 \%$ probability of observing that failure.

\section{Protocol}

The protocol was reviewed and validated by a national ethics committee according to French and European directives, with written consent from the patients.

\section{Inclusion}

1. Include patients with resectable CLM and a good clinical condition (Eastern Cooperative Oncology Group ECOG/World Health Organization WHO performance status $0-1$ ) who are at least 18 years old. Obtain written consent from the patients.

2. Exclude patients with previous liver, biliary, or major abdominal surgery, pregnancy, or an inability to be followed for the duration of the study.

\section{HIFU Treatment}

1. Perform the anesthesia of the patient with an induction composed of propofol ( 2 to $3 \mathrm{mg} / \mathrm{kg}$ ), sufentanil $(0.2$ to $0.3 \mu \mathrm{g} / \mathrm{kg})$, cisatracurium ( 0.15 $\mathrm{mg} / \mathrm{kg}$ ) and ketamine $(0.15 \mathrm{mg} / \mathrm{kg})$.

2. Maintain anesthesia with sevoflurane to target a bispectral index between 40 and 60 . 
3. Perform tracheal intubation, with the lungs mechanically ventilated with inspired oxygen fraction of $80 \%$.

4. Prepare the HIFU device in the operating room by covering the sterilized HIFU probe with the dedicated sterile envelope containing a sterile ultrasound coupling liquid (Figure 1).

NOTE: The HIFU probe was sterilized using a low-temperature gas plasma sterilizer.

5. Perform laparotomy using a monopolar knife after classic surgical asepsis.

6. Place the retractors under the rib cage and between the edges of the surgical incision.

7. Confirm liver resection by exploring the liver using intraoperative diagnostic ultrasound images with a $7.5 \mathrm{MHz} \mathrm{T-type} \mathrm{transducer} \mathrm{and}$ palpation to confirm that no additional metastasis compromising the resection was missed with preoperative imaging ${ }^{22}$.

8. Free the liver from its peritoneal and vascular attachments with scissors.

9. Place a protective abdominal gauze sponge $\left(20 \times 20 \mathrm{~cm}^{2}\right)$ with X-ray detectable threads under the targeted area to inhibit ultrasound propagation and unintended HIFU damage to adjacent organs.

10. Bring the HIFU probe in contact with the liver surface (Figure 2).

11. Move the HIFU probe on the surface of the liver until the position of the expected HIFU ablation (represented by the white cone superimposed on the sonogram) is at the right location according to the objectives of the different phases (Figure 3a).

12. Activate the HIFU exposure by activating each emitter with the same phase for $40 \mathrm{~s}$. Apply an acoustic power of $70 \mathrm{~W}$ (free-field acoustic power) for superficial ablations and $90 \mathrm{~W}$ (free-field acoustic power) for in-depth lesions.

1. Ultrasound images were not synchronized with HIFU exposures and, therefore, interferences were visible in sonograms during ablations. Observe that boiling is created in liver tissues treated during HIFU single exposures. Note that a cloud of ebullition appears momentarily for $1 \mathrm{~min}$ at the location of the lesion (Figure $\mathbf{3 b}$ ).

NOTE: The single lesion appears on the sonogram as a hyperechoic zone at its center and a hypoechoic zone at its boundaries (Figure 4a).

13. Mark the position of the HIFU probe, mainly the acoustic axis at the surface of the liver, using a surgical marker, to know where to cut the liver for gross examination (Figure 2).

14. Measure the dimensions of the HIFU ablations along two orthogonal planes after each HIFU exposure, using the integrated ultrasound imaging probe.

15. Move the HIFU probe on the liver surface to confirm that all eight liver segments can be covered, irrespective of their conformation in the individual patient.

\section{Macroscopical and Histological Evaluation of HIFU Lesions}

1. Perform the planned liver resection using a Kelly clamp to crush the liver parenchyma and a scissor to cut the liver. Perform hemostasis with titanium clips and/or irrigating bipolar forceps.

2. Close the aponeurosis with monofilament, long-lasting absorbable $\mathrm{N}^{\circ} 0$ or $\mathrm{N}^{\circ} 1$ suture material.

3. Close the skin with uncolored, short-term absorbable $\mathrm{N}^{\circ} 3 / 0$ suture material.

4. Give the patient oral acetaminophen ( $1 \mathrm{~g}$ every $6 \mathrm{~h}$ ).

NOTE: Nausea and vomiting were treated with $4 \mathrm{mg}$ of intravenous ondansetron. Oral fluids and feeding were started on the day after surgery. Morphine was given to obtain a pain visual analogic scale under 30/100.

5. Separate the HIFU ablations and areas of CLM with a scalpel.

6. Cut the ablations along the exposure axis with a scalpel.

7. Take pictures of the HIFU ablations.

8. Measure the dimensions of the ablated area macroscopically with a ruler and on a photograph, using appropriate image analysis software.

9. Slice each ablation into $5 \mathrm{~mm}$ sections to assess macroscopic homogeneity.

10. Fix the ablated areas in $10 \%$ phosphate-buffered formalin $(\mathrm{pH}=7)$, embed them in paraffin, and stain them with hematoxylin and eosin.

11. Histologically examine the ablated and surrounding tissues.

\section{Follow-up}

1. Follow the patients for one month according to the institution's guidelines for patients resected for CLM.

2. Summarize data regarding demographic characteristics, vital signs (baseline and 5 min after HIFU), and safety observations and measurements regarding the HIFU lesions.

3. Use Spearman's rank correlation coefficient to test the association between variables.

\section{Representative Results}

Fifteen patients were included between March 2010 and November 2011. Six patients were included in phase I. Six patients were included in step 1 of phase lla. Three patients were included in step 2 of phase lla. A right or extended right hepatectomy was performed in all patients. Phase IIb is currently ongoing, and results are not described. Complete results of phases I and Ila have been previously published ${ }^{14}$. 


\section{Phase I:}

In total, twelve HIFU ablations were successfully created in the six phase-I patients (Figure 5). One superficial HIFU ablation and one deep HIFU ablation were created in each of the 6 patients. Deep HIFU lesions were placed at a mean depth of $12 \pm 2$ (10-20) mm corresponding to a focal distance of $22 \pm 2(20-40) \mathrm{mm}$ (Figure 3a). The resulting mean distance between Glisson's capsule and the HIFU ablation was, on average, $7 \pm$ $4(2-13) \mathrm{mm}$. Asepsis was not comprised in all patients during the preparation and utilization of the device. There was no lesion in neighboring tissues after HIFU ablation. There were no any substantial changes in respiratory and hemodynamic parameters. The duration of the HIFU procedure was, on average, $15 \pm 7(9-27) \mathrm{min}$. The ultrasound imaging probe integrated with the HIFU device allows visualizing, on average, $88 \%$ (95\% Cl: 67 to $100 \%)$ of the 15 predefined areas.

\section{Phase lla:}

For the first three patients of step 1, the metallic mark had not been included in the HIFU lesions in one of six ablations. After reviewing the record of the procedure, it was established that a Glissonian pedicle was confused with the metallic marker (Figure 6). As required by the study design, three more patients were included, and all six additional HIFU lesions were correctly created around all six metallic markers. Since the primary objective of step 1 was achieved, the steering committee approved the continuation of the study.

The aim of step 2 was to achieve ablations distant from a metallic marker by $7.5 \mathrm{~mm}$. Six ablations with a distance of $7.0 \pm 2.3(4.3-9.8) \mathrm{mm}$ were achieved, demonstrating that it was possible to preserve an area from damage. The procedure was safe, asepsis was not compromised, and there was no lesion in the neighboring organs and no rupture of Glisson's capsule.

\section{Common Secondary Objectives of Phases I and Ila:}

All 30 HIFU ablations created in 15 patients were slightly cone-shaped. The average length of the HIFU ablations was $27.5 \pm 6 \mathrm{~mm}$. The average superior width of the HIFU ablations was $21 \pm 3.9 \mathrm{~mm}$. In ultrasound images, the average length of HIFU ablations was $28.9 \pm 4.6 \mathrm{~mm}$ and the average superior width was $23.9 \pm 3.8 \mathrm{~mm}$. Figure $\mathbf{4 b}$ shows the rank correlation coefficient $(r=0.88 ; p<0.001 ; 95 \% \mathrm{Cl}, 0.82$ to 0.91$)$ between macroscopic measurements and measurements performed using ultrasound images.

Figure 7 shows the capability of the imaging system to visualize metastases. Figure $7 a$ and $7 \mathrm{c}$ show examples of two metastases imaged using the ultrasound imaging probe integrated with the HIFU device. Figure $\mathbf{7 b}$ and $\mathbf{7 d}$ show the corresponding metastases observed on gross pathology after surgery. The HIFU focal region was superimposed on the sonogram and displayed in the user interface, making it possible to place the location of the ablation with precision (Figure 7a).

As illustrated in Figure 8, the delimitation between treated and untreated liver is very sharp (about $200 \mu \mathrm{m}$ ). There was a halo of congestive tissues around all HIFU ablations. The microscopic examination confirmed homogeneous necrosis with all HIFU ablations. Since there were only 1 to $2 \mathrm{~h}$ between HIFU ablation and hepatectomy, it was not possible to observe evidence of apoptosis. However, pronounced alterations in hepatocytes were observed in all HIFU ablations. The microscopic analysis confirmed homogeneous ablations with all cases, notably around vessels (Figure 8).

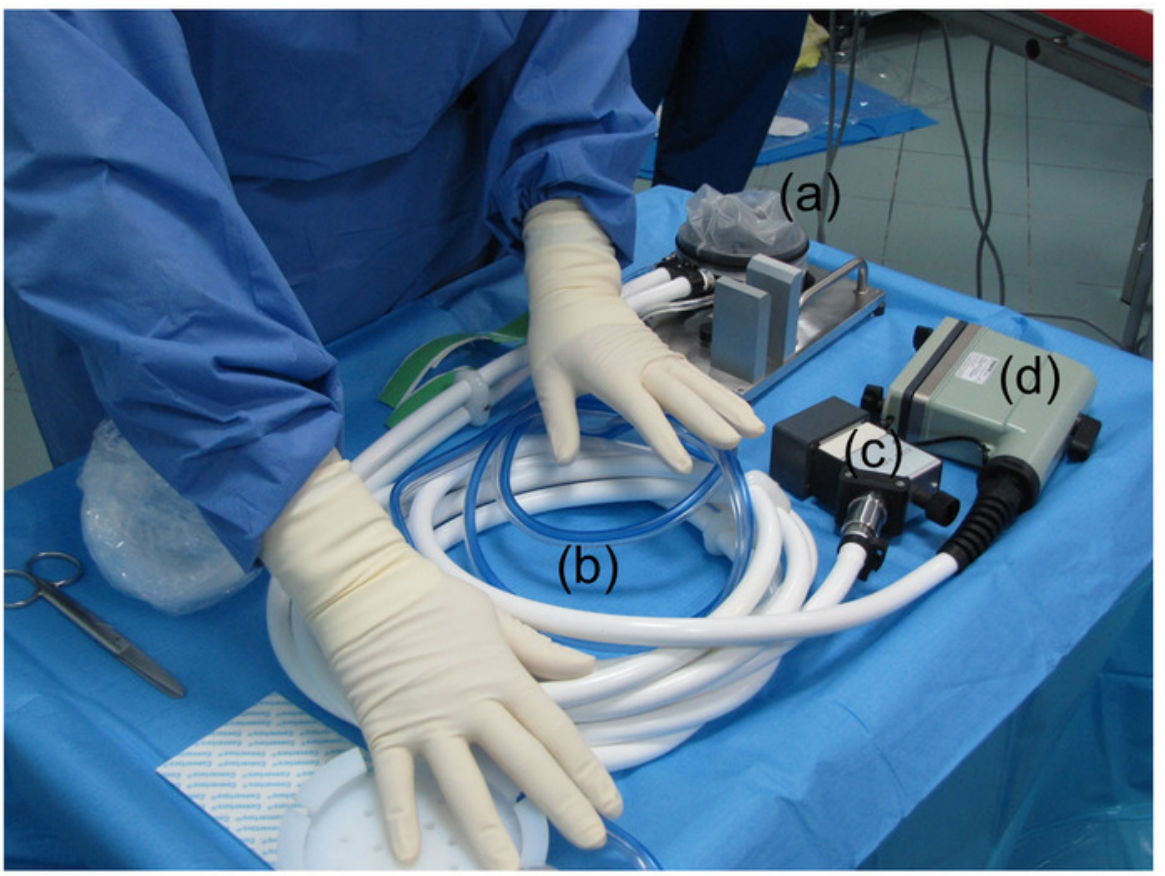

Figure 1: Preparation of the HIFU device. The HIFU device was prepared by covering the sterilized HIFU probe with a sterile envelope containing an ultrasound coupling liquid. (a) This panel shows the HIFU transducer covered with a sterile envelope. (b) This panel shows the tube for the cooling circuit. (c) This panel shows the connector for the electrical power. (d) This panel shows the connector for the ultrasound imaging probe. Please click here to view a larger version of this figure. 


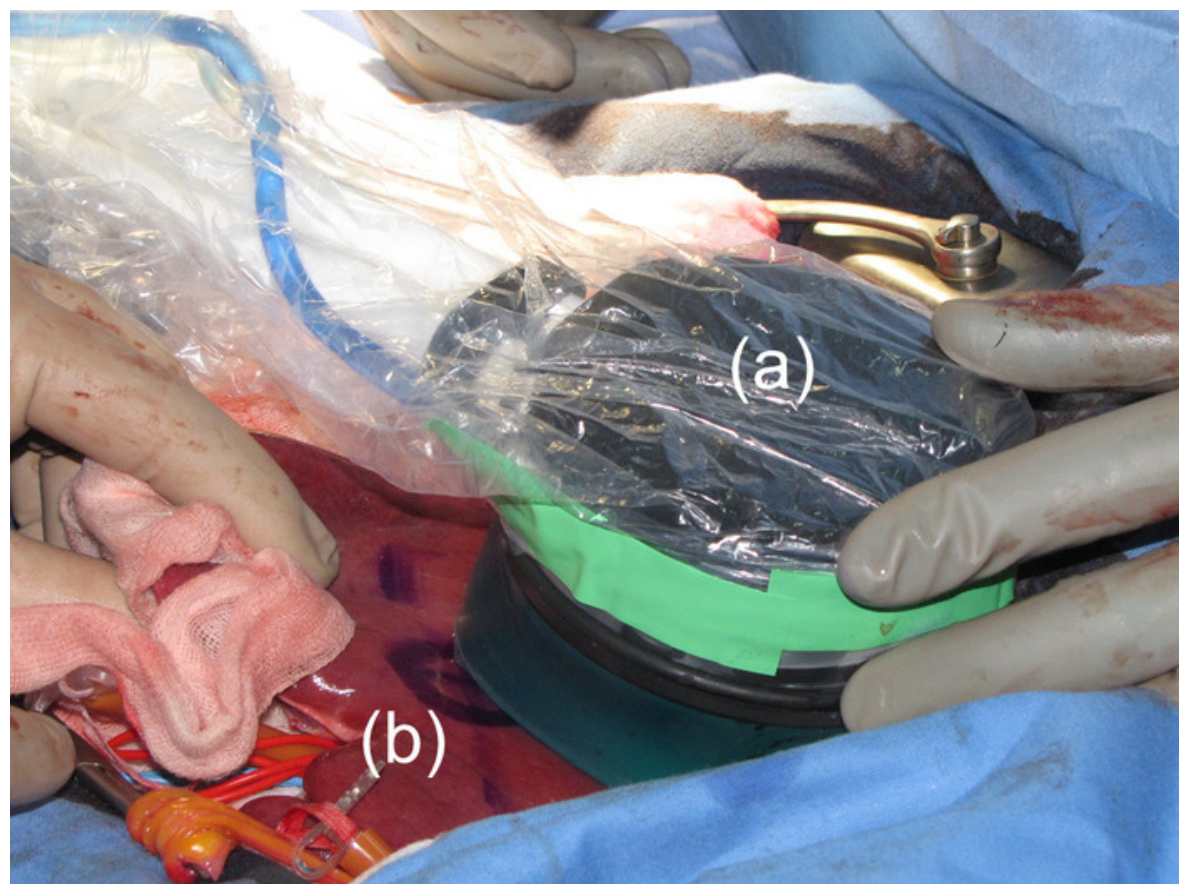

Figure 2: Use of the HIFU probe. The HIFU probe was held by hand and brought into contact with the liver surface. (a) This panel shows the HIFU probe. (b) The position of the HIFU probe at the time of ablation was marked on the liver. Please click here to view a larger version of this figure.

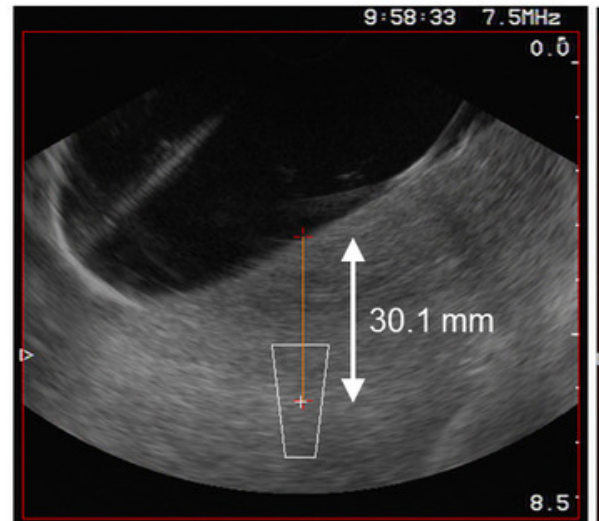

(a)

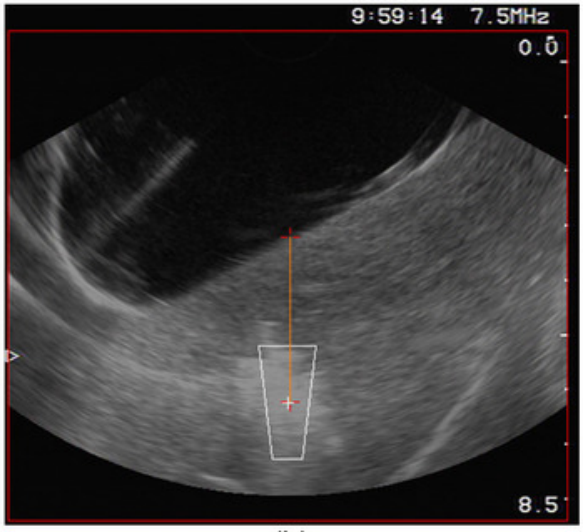

(b)

Figure 3: Targeting process. (a) The user interface displayed the position of the HIFU focal region superimposed on the sonogram, making it possible to place the location of the ablation in the tissues. (b) Immediately after the HIFU exposure, a cloud of ebullition appeared momentarily (for approximately $1 \mathrm{~min}$ ) at the location of the lesion. Please click here to view a larger version of this figure. 

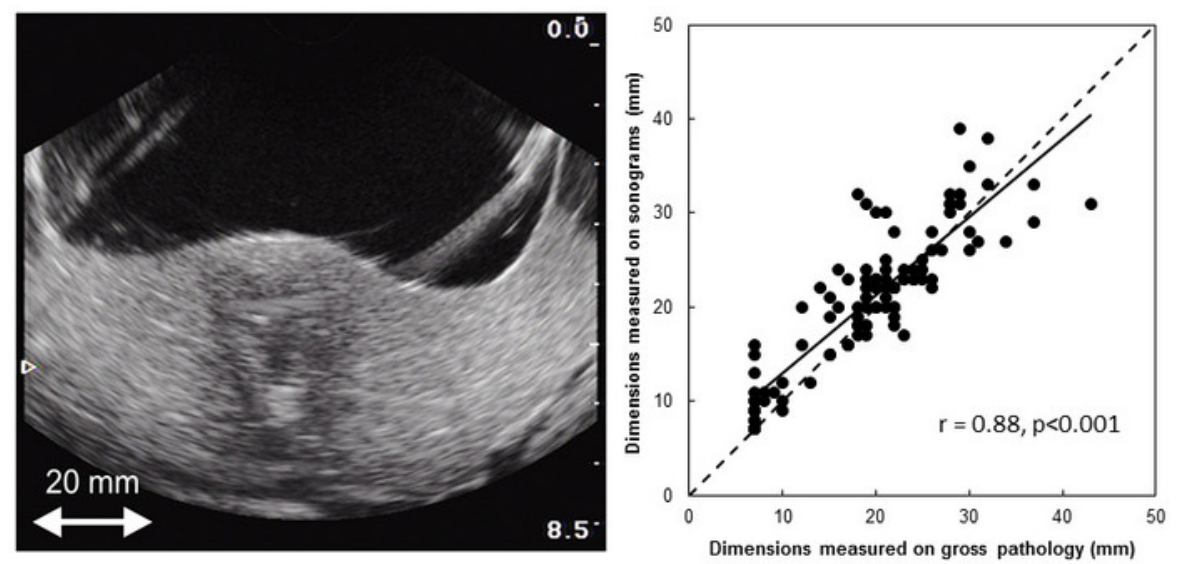

Figure 4: Monitoring. After boiling, the single lesion appeared on the sonogram as a hyperechoic zone at its center and a hypoechoic zone at its boundaries. Please click here to view a larger version of this figure.

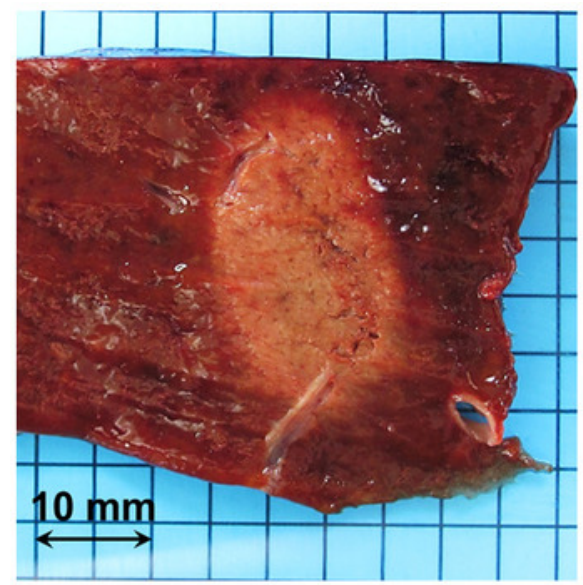

(a)

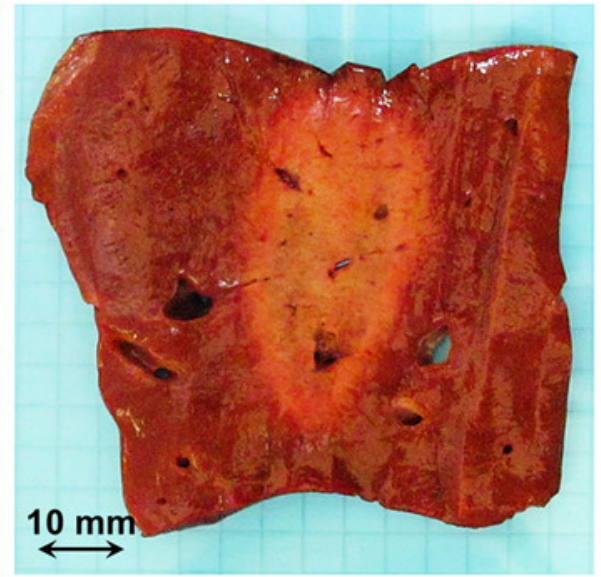

(b)

Figure 5: Macroscopic visualization of two HIFU ablations. HIFU ablations were clearly visible by gross pathology. (a) This panel shows a typical HIFU ablation. (b) This panel shows an example of a HIFU lesion created during step 1 of phase Ila. The aim was to target the metallic marker. The shape and homogeneity of the ablation were not altered by the presence of hepatic veins. Please click here to view a larger version of this figure. 


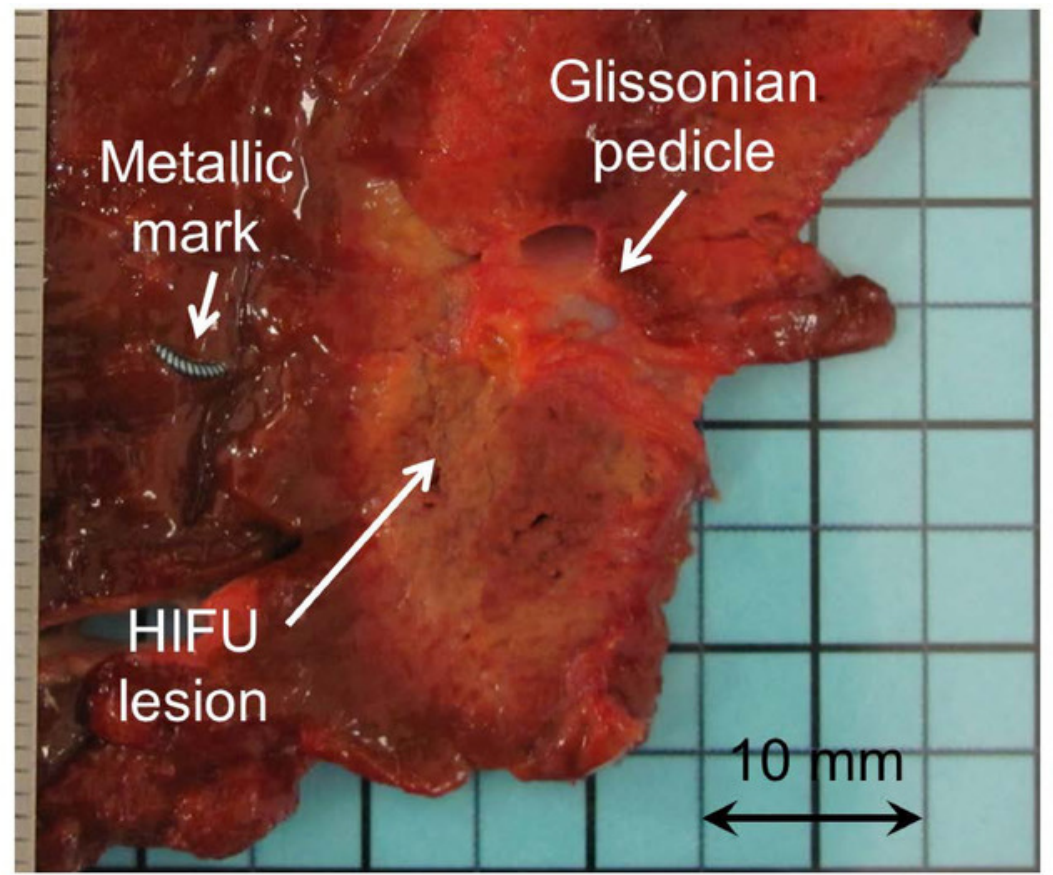

Figure 6: Macroscopic visualization of a HIFU ablation created in phase Ila. In one case, the metallic marker had not been included in the HIFU. After reviewing the record of the procedure, it was established that a Glissonian pedicle was confused with the metallic marker. Please click here to view a larger version of this figure. 


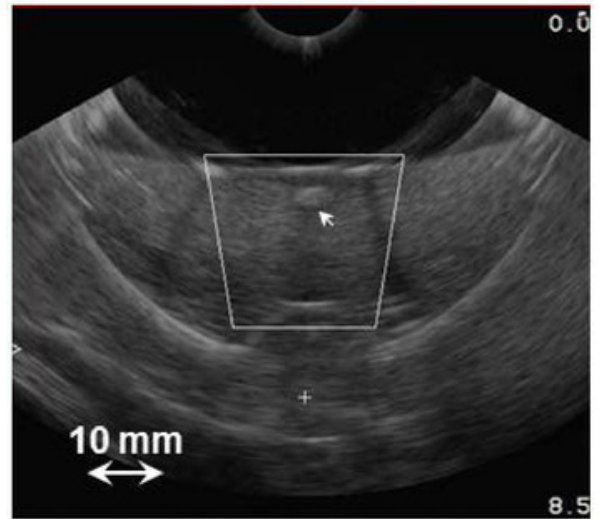

(a)

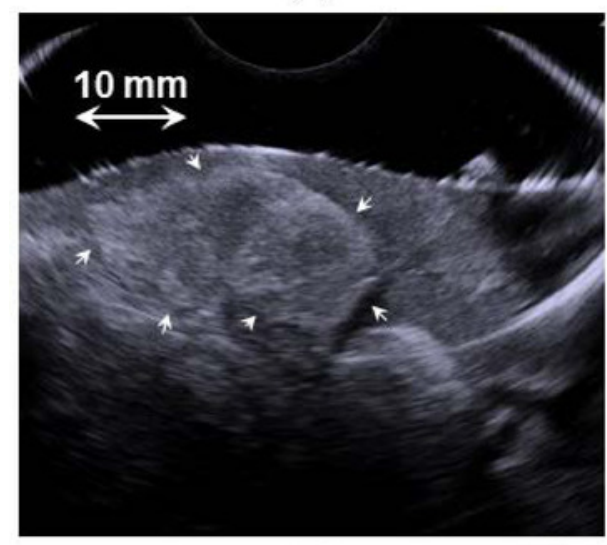

(c)

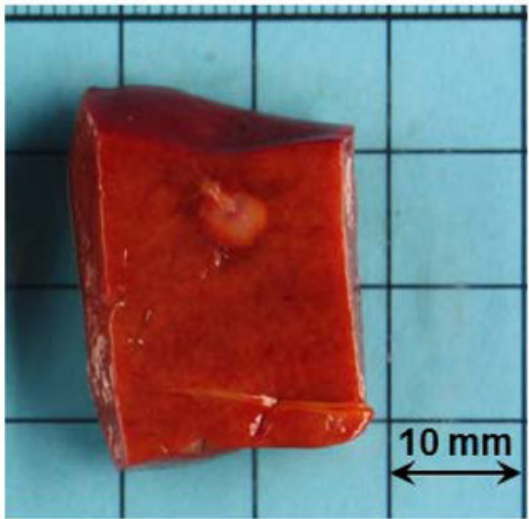

(b)

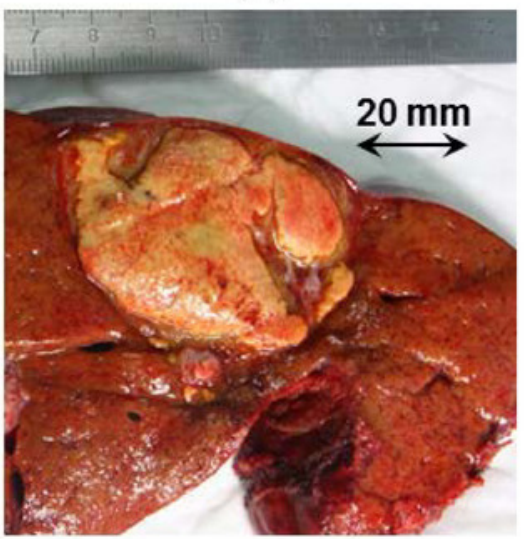

(d)

Figure 7: Visualization of metastases using the integrated ultrasound imaging probe. Panels (a) and (c) show examples of two metastases imaged using the ultrasound imaging probe integrated with the HIFU device. Panels (b) and (d) show the corresponding metastases observed by gross pathology after surgery. (a) The user interface displayed the position of the HIFU focal region superimposed on the sonogram, making it possible to place the location of the ablation in the tissues and to visualize if the metastasis will be treated with safety margins. Please click here to view a larger version of this figure. 


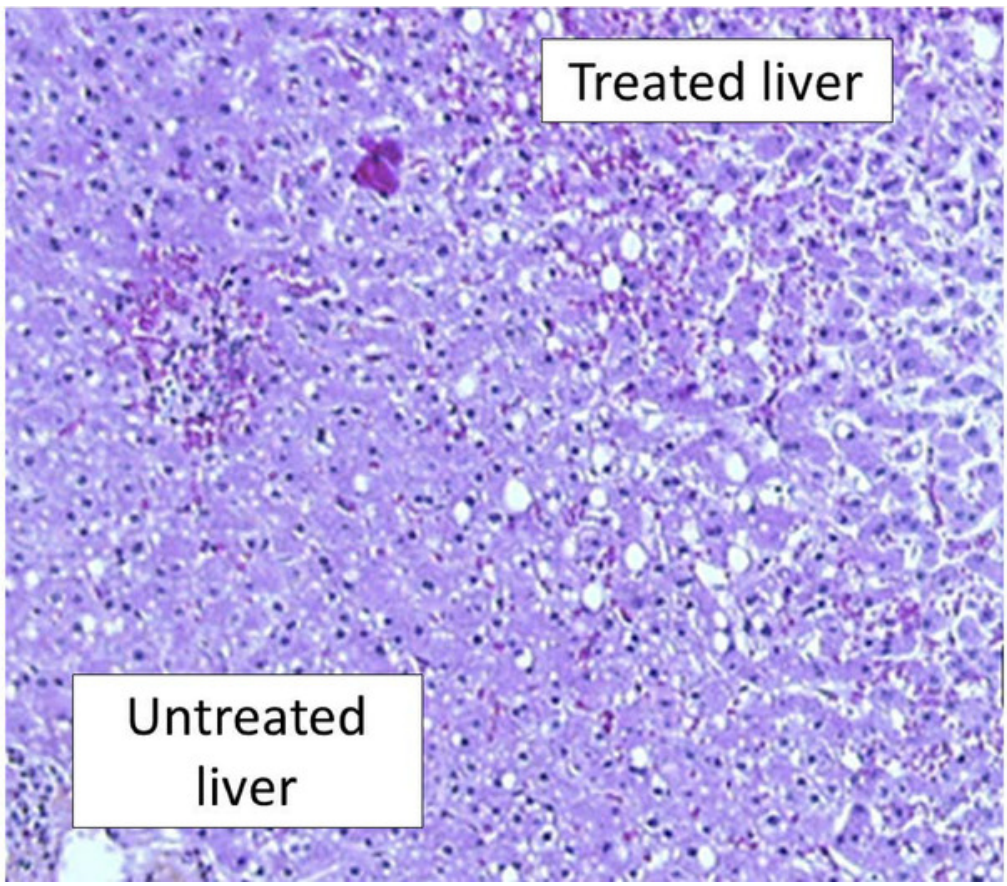

Figure 8: Histopathological examination after HIFU ablation. This panel shows a representative liver examination using H\&E staining. There was a sharp demarcation between treated and untreated liver with pronounced alterations in hepatocytes. Here, the magnification is $200 x$. Please click here to view a larger version of this figure.

\section{Discussion}

The aim of this study was to confirm the feasibility, safety, and efficacy of a new intraoperative HIFU device. As this was its first use in humans, an ablate-and-resect study was designed in order to verify the ability of the device to create complete ablation and accurate targeting without compromising the chance of potentially curative therapy for patients. In total, $30 \mathrm{HIFU}$ ablations were safely created in 15 patients, each within $40 \mathrm{~s}$. The average dimensions of the HIFU ablations were $27.5 \times 21.0 \mathrm{~mm}^{2}$. There was no secondary lesion in neighboring organs. Importantly, the shape of the HIFU ablations was not altered by the major blood vessels (up to $5 \mathrm{~mm}$ in diameter). This is of particular importance since it has been reported that blood flow plays a pivotal role in thermal ablation by limiting the ablation size and altering ablation shape ${ }^{23}$. Another important advantage of this intraoperative HIFU device when compared with ablations performed using radiofrequency or microwaves is that there is no need to insert a probe in the liver parenchyma.

The choice of developing a HIFU device dedicated to intraoperative use was supported by oncological necessities and technological challenges that remain unmet in HIFU technology. Extracorporeal HIFU treatments have been proved to be clinically feasible. However, the liver volume accessible to an extracorporeal HIFU treatment is about $30 \%$ unless a partial rib resection is performed ${ }^{24}$. In addition, when using HIFU extracorporeally for treating liver tumors, skin burns and gastric lesions can be created ${ }^{25,26}$. The intraoperative approach was selected to avoid these potentially important complications.More importantly, intraoperative ablations are widely employed in the management of CLM, whether they are associated with surgery or not. Despite improvements in imaging, new liver metastasis are found in approximately $15 \%$ of patients ${ }^{22}$, and new findings, such as peritoneal carcinomatosis, may preclude the need for liver-directed therapy. For this first use in humans of the intraoperative HIFU device, ablations were created close to metastases and only in areas planned for resection. This first step was regarded as essential to demonstrate the accuracy and the safety of this new HIFU device before considering its routine use to treat CLM.

The size of the HIFU probe $(10 \mathrm{~cm}$ in diameter) does not limit its ease of use since it was possible to place the probe as such that it allowed a targeting of approximately $90 \%$ of the entire liver. This was possible even through a limited midline laparotomy. HIFU ablations were easily identified by ultrasound imaging, allowing a reliable monitoring of the treatment, which is a major advantage over other ablative techniques. The slight difference between ablation dimensions measured on ultrasound images and those measured on macroscopic examination was due to the difficulty to cut fresh liver exactly along the imaging plane.

Although this study confirmed the theoretical advantages of HIFU for liver ablation (i.e., no need for an intraparenchymal introduction of a probe and real-time monitoring, and independence from the heat-sink effect), the efficacy of intraoperative HIFU should be demonstrated on liver metastases. If the ability to target liver metastases is confirmed during phase Ilb, the next step will be to design a multicenter prospective cohort of patients treated with curative-intent with intraoperative HIFU.

\section{Disclosures}

The authors have nothing to disclose. 


\section{Acknowledgements}

This work was partly funded by the LYriCAN (INCa-DGOS-Inserm_12563). The authors acknowledge the Claude Bernard University Lyon 1 for the award of the Antonin Poncet prize for work related to this study. The authors gratefully acknowledge the willing participation of the patients involved, EDAP-TMS, the role of the Steering committee (Dominique Elias, Olivier Rouviere, Alain Sezeur, Frédéric Marchal), which consisted of responsibility for deciding on the continuation of the study after an interim analysis, as well as Jean-Yves Scoazec for pathological examination.

\section{References}

1. Nordlinger, B. et al. Perioperative FOLFOX4 chemotherapy and surgery versus surgery alone for resectable liver metastases from colorectal cancer (EORTC 40983): long-term results of a randomised, controlled, phase 3 trial. The Lancet Oncology. 14 (12), 1208-1215 (2013).

2. Adam, R., Laurent, A., Azoulay, D., Castaing, D., Bismuth, H. Two-stage hepatectomy: A planned strategy to treat irresectable liver tumors. Annals of Surgery. 232 (6), 777-785 (2000).

3. Folprecht, G., Grothey, A., Alberts, S., Raab, H. R., Kohne, C. H. Neoadjuvant treatment of unresectable colorectal liver metastases: correlation between tumour response and resection rates. Annals of Oncology. 16 (8), 1311-1319 (2005).

4. Pawlik, T. M., Schulick, R. D., Choti, M. A. Expanding criteria for resectability of colorectal liver metastases. The Oncologist. 13 (1), 51-64 (2008).

5. Kennedy, J. E. High-intensity focused ultrasound in the treatment of solid tumours. Nature Reviews Cancer. 5 (4), $321-327$ (2005).

6. Uchida, T. et al. High-intensity focused ultrasound therapy for prostate cancer. International Journal of Urology. 19 (3), 187-201 (2012).

7. Crouzet, S. et al. Whole-gland ablation of localized prostate cancer with high-intensity focused ultrasound: oncologic outcomes and morbidity in 1002 patients. European Urology. 65 (5), 907-914 (2014).

8. Haar, G. T., Coussios, C. High intensity focused ultrasound: physical principles and devices. International Journal of Hyperthermia. 23 (2), 89-104 (2007).

9. van Amerongen, M. J., Jenniskens, S. F. M., van den Boezem, P. B., Futterer, J. J., de Wilt, J. H. W. Radiofrequency ablation compared to surgical resection for curative treatment of patients with colorectal liver metastases - a meta-analysis. HPB (Oxford). 19 (9), $749-756$ (2017).

10. Lin, Z. Y. et al. Effect of heat sink on the recurrence of small malignant hepatic tumors after radiofrequency ablation. Journal of Cancer Research and Therapeutics. 12 (Supplement), C153-C158 (2016).

11. Stoltz, A., Gagniere, J., Dupre, A., Rivoire, M. Radiofrequency ablation for colorectal liver metastases. Journal of Visceral Surgery. 151 Suppl 1, S33-44 (2014).

12. Vogl, T. J. et al. Evaluation of microwave ablation of liver malignancy with enabled constant spatial energy control to achieve a predictable spherical ablation zone. International Journal of Hyperthermia. 34 (4), 492-500 (2018).

13. Shady, W. et al. Percutaneous Microwave versus Radiofrequency Ablation of Colorectal Liver Metastases: Ablation with Clear Margins (A0) Provides the Best Local Tumor Control. Journal of Vascular and Interventional Radiology. 29 (2), 268-275 e261 (2018).

14. Dupre, A. et al. First Clinical Experience of Intra-Operative High Intensity Focused Ultrasound in Patients with Colorectal Liver Metastases: A Phase I-Ila Study. PLoS One. 10 (2), e0118212 (2015).

15. Aubry, J. F. et al. The road to clinical use of high-intensity focused ultrasound for liver cancer: technical and clinical consensus. Journal of Therapeutic Ultrasound. 1, 13 (2013).

16. Melodelima, D., N'Djin, W. A., Parmentier, H., Chesnais, S., Rivoire, M., Chapelon, J. Y. Ultrasound surgery with a toric transducer allows the treatment of large volumes over short periods of time. Applied Physics Letters. 91 (19), 193901 (2007).

17. N'Djin, W. A. et al. Utility of a tumor-mimic model for the evaluation of the accuracy of HIFU treatments. results of in vitro experiments in the liver. Ultrasound in Medicine \& Biology. 34 (12), 1934-1943 (2008).

18. Melodelima, D. et al. Thermal ablation produced using a surgical toroidal high-intensity focused ultrasound device is independent from hepatic inflow occlusion. Physics in Medicine \& Biology. 54 (20) (2009).

19. Melodelima, D. et al. Thermal ablation by high-intensity-focused ultrasound using a toroid transducer increases the coagulated volume. Results of animal experiments. Ultrasound in Medicine \& Biology. 35 (3), 425-435 (2009).

20. Parmentier, H. et al. High-intensity focused ultrasound ablation for the treatment of colorectal liver metastases during an open procedure: study on the pig. Annals of Surgery. 249 (1), 129-136 (2009).

21. Lee, Y. J., Wesley, R. A. Statistical contributions to phase II trials in cancer: interpretation, analysis and design. Seminars in Oncology. 8 (4), 403-416 (1981).

22. Arita, J. et al. Routine Preoperative Liver-specific Magnetic Resonance Imaging Does Not Exclude the Necessity of Contrast-enhanced Intraoperative Ultrasound in Hepatic Resection for Colorectal Liver Metastasis. Annals of Surgery. 262 (6), 1086-1091 (2015).

23. Vahldiek, J. L. et al. Measuring and optimizing results in multipolar RFA: Techniques and early findings in an experimental setting. Clinical Hemorheology and Microcirculation. 58 (1), 77-87 (2014).

24. Zhu, H. et al. High intensity focused ultrasound (HIFU) therapy for local treatment of hepatocellular carcinoma: role of partial rib resection. European Journal of Radiology. 72 (1), 160-166 (2009).

25. Tanter, M. et al. Compensating for bone interfaces and respiratory motion in high-intensity focused ultrasound. International Journal of Hyperthermia. 23 (2), 141-151 (2007).

26. Jung, S. E., Cho, S. H., Jang, J. H., Han, J. Y. High-intensity focused ultrasound ablation in hepatic and pancreatic cancer: complications. Abdominal Radiology. 36 (2), 185-195 (2011). 